\title{
Experimental Analyses of Load Carrying Effects on the Peak Traction Coefficient between Shoe Sole and Floor during Walking
}

\author{
Takeshi Yamaguchi ${ }^{*}$, Hiroteru Yamanouchi and Kazuo Hokkirigawa \\ Graduate School of Engineering, Tohoku University \\ 6-6-01 Aramaki-Aza-Aoba, Aoba-ku, Sendai, Miyagi 980-8579, Japan \\ *Corresponding author: yamatake@cc.mech.tohoku.ac.jp
}

( Manuscript received 26 September 2008; accepted 10 November 2008; published 30 November 2008 )

\begin{abstract}
This paper presents the effect of load carrying (position and weight of load) on the maximum peak values of traction coefficient between shoe sole and floor during walking on level walkway. Gait experiments were conducted by five healthy male adult participants walking on the force plates with carrying the loads. Each subject was tested at step length of $0.75 \mathrm{~m}$ and at walking speed of $1.4 \mathrm{~m} / \mathrm{s}$. They were instructed to carry the loads by three different ways; a 2-handed load carrying, a back-pack load carrying, and a waist level front load carrying. Weight of load was 49, 98, 147 or $196 \mathrm{~N}$ for each way of load carrying. For a waist level front load carrying, the maximum peak traction coefficient at heel strike period $\left|F_{h} / F_{h}\right|_{h}$ statistically significantly ( $p$ $<0.01)$ decreased with increasing of weight of load. On the other hand for other ways of load carrying, $\left|F_{h} / F_{n}\right|_{h}$ was not affected significantly $(p>0.05)$ by weight of load. For each way of load carrying, the maximum peak value of traction coefficient at toe-off period $\left|F_{h} / F_{n}\right|_{t}$ had tendency to increase with increase of weight of load. The relationship between the slip potential and the COM position change by load carrying were also discussed.
\end{abstract}

Keywords: fall, slip, traction coefficient, load carrying, body center of mass, kinematics, tribology

\section{Introduction}

Recently, a percentage of falling accidents in the occupational accidents has been increasing in Japan as well as in other countries. About $20 \%$ in Japan (2004) ${ }^{1)}$ and $12 \%$ in the USA $(1998)^{2)}$ of the number of casualties in the occupational accidents were caused by falling accidents. In the U.S., it is reported that a majority of the work-related falls attributed to slipping. Furthermore, it is also found that more than $30 \%$ of injuries while load carrying had a slip or a trip as a contributing factor ${ }^{3)}$. Thus, it is considered very important to understand load carrying effects on the slip potential between shoe sole and floor to prevent such slip-related falling accidents.

Cham and Redfern ${ }^{3)}$ found that the peak traction coefficient between shoe sole and floor at heel-strike period, a variable to rate the slip potential, was affected by load carrying. They clarified experimentally that the peak traction coefficient reduced during walking with carrying the loaded crate at about waist level using the 2-handed symmetric posture as compared with walk without load. Yamaguchi et al. ${ }^{4}$ found that the peak traction coefficients at both heel-strike and toe-off periods while waking without carrying loads can be well estimated by the geometric relationship between the whole body center of mass (COM) and the foot center of pressure (COP) during walking. The foot COP corresponds to the point of application of the ground reaction force. It would be considered that the position of the whole body COM changes by the load position and weight of load while walking with carrying loads, which would result a variation of the peak traction coefficients.

Thus, the purpose of this study was to investigate the effects of load carrying, position and weight of load, on the maximum peak traction coefficients at heel-strike and toe-off periods between shoe sole and floor during walking on level walkway. The relationship between the slip potential and the change of COM position by the position and weight of loads was also discussed.

\section{Methods}

\subsection{Subjects}

Five male adults between the ages of 21-30 years with no musculoskeletal, neurological or gait abnormalities participated in this study. Their weight ranged from 59.5 to $86.5 \mathrm{~kg}$ (mean: $67.9 \mathrm{~kg}$, SD: 11.2 $\mathrm{kg}$ ), the body stature ranged from 177 to $182 \mathrm{~cm}$ (mean: $177.5 \mathrm{~cm}$, SD: $3.5 \mathrm{~cm}$ ). Informed consent was obtained 
from each subject prior to participation.

\subsection{Experimental set-up}

Figure 1(a) shows experimental set-ups for gait experiment. Force plate array consisted of eight force plates (OR6-5, Advanced Mechanical Technology Inc.) were used to collect three orthogonal components of the ground reaction force $\left(F_{x}, F_{y}, F_{n}\right)$ as shown in Fig. 1(b). The gait experiments were carried out under dry level walkway condition. An indoor carpet was used as floor material. Full body kinematics was recorded using a three dimensional motion capture system (vicon 612, Vicon Motion Systems Ltd.). Thirty-four infrared-reflective markers were attached to bilateral upper and lower extremities and a torso of each subject. Commercially available men's shoes were used, and flat NBR (nitrile butadiene rubber, thickness $1.8 \mathrm{~mm}$, shore hardness HS70) sheets affixed to the shoe bottom served at the shoe sole in order to eliminate the influence of shoe sole patterns on the ground reaction force.

\subsection{Experimental condition}

Each subject was tested at step length of $0.75 \mathrm{~m}$ and at walking speed of $1.4 \mathrm{~m} / \mathrm{s}$. These values of step length and walking speed were selected based on the preferred step length and walking speed in Japanese male in twenties reported by Sekiya et al. ${ }^{5)}$. The step length was standardized by stepping on marks on the walkway, and the cadence was standardized by using a metronome to standardize walking speed. The walking start-point located $1.5 \mathrm{~m}$ before the force plates, thus, the ground reaction forces in this study were collected from steady state walking not from transient walking.

The subjects were instructed to carry the loads by three different ways as shown in Fig. 2; carrying a dumbbell in each hand (Fig. 2(a); 2-handed load carrying), carrying a disk-shaped load on their back (Fig. 2(b); back-pack load carrying) and carrying the disk-shaped load at about waist level using the 2-handed symmetrical posture (Fig. 2(c); waist level front load carrying). Weight of load was 49, 98, 147 or $196 \mathrm{~N}$. They were also asked to walk as stepping with left foot on the left side of force plates and stepping with the right foot on the right side of force plates. The infrared-reflective markers were attached to the ends of the dumbbell shaft and the center of disk-shaped loads as shown in Fig. 2 for calculation of the position of the center of mass of each load $\left(\mathrm{COM}_{\mathrm{L}}\left(x_{C O M L}, y_{C O M L}\right.\right.$, $\left.z_{\text {СOML }}\right)$ ). The ground reaction force data of left foot and right foot ( 2 steps) on the left and right side of force plates were captured for each condition, and the subjects walked 3 times under the same walking condition.

\subsection{Data analysis}

The traction coefficient was defined as the absolute value of the horizontal ground reaction force divided by the normal ground reaction force $\left(\left|F_{h} / F_{n}\right|\right)$. The period from heel contact to whole shoe-sole contact (foot flat) was the heel-strike period; the period from heel-off to

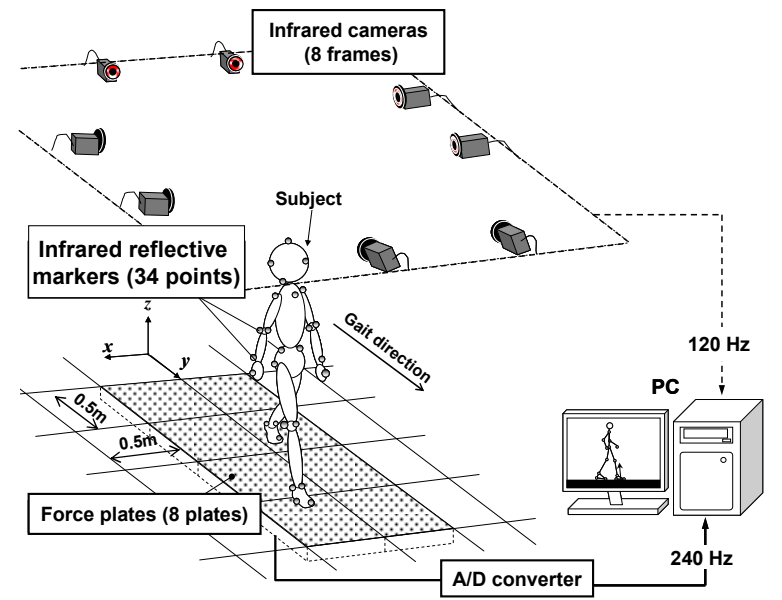

(a)

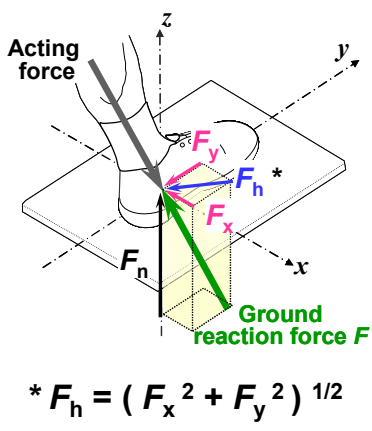

(b)

Fig. 1 Schematic diagram of (a) experimental set-up of gait experiments and (b) orthogonal components of ground reaction force

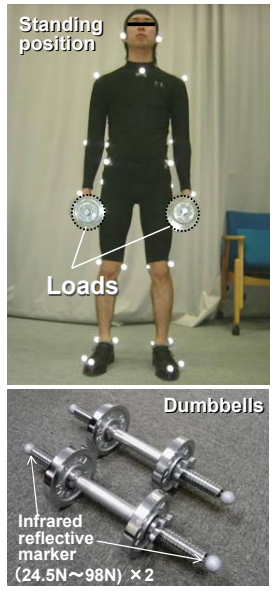

(a)

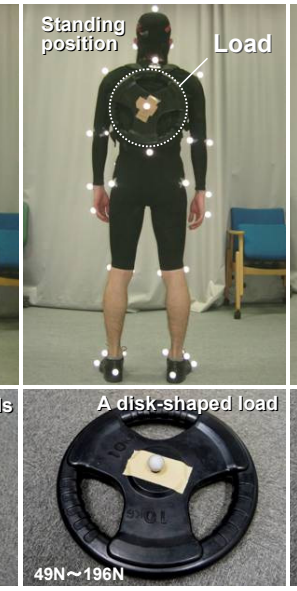

(b)

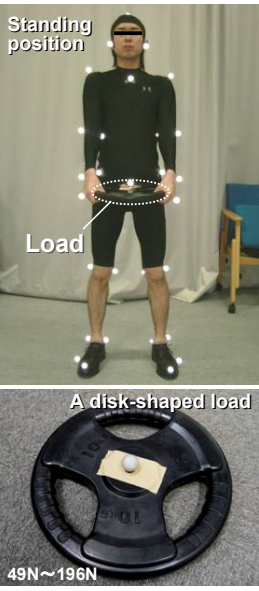

(c)
Fig. 2 Ways of load carrying. (a) 2-handed load carrying, (b) back-pack load carrying, (c) waist level front load carrying

toe-off was the toe-off period.

The whole body center of mass (COM) position $\left(x_{C O M}, y_{C O M}, z_{C O M}\right)$ was computed from the kinematic data using known Japanese segmental parameters ${ }^{6)}$ in 14-segment configurations consisting the head, trunk, bilateral upper arm, bilateral forearm, bilateral hand, 
bilateral thigh, bilateral shank and bilateral foot. A position of the integrated COM $\left(\mathrm{COM}_{\mathrm{i}}\left(x_{\text {COMi }}, y_{\text {COM } i}\right.\right.$, $\left.z_{C O M i}\right)$ ) which is the position of the body center of mass including loads was calculated by using following equations;

$$
\begin{aligned}
& x_{\mathrm{COM}_{i}}=\frac{B M \cdot x_{\mathrm{COM}}+m \cdot x_{\mathrm{COM}_{L}}}{B M+m} \\
& y_{\mathrm{COM}_{i}}=\frac{B M \cdot y_{\mathrm{COM}}+m \cdot y_{\mathrm{COM}}}{B M+m} \\
& z_{\mathrm{COM}_{i}}=\frac{B M \cdot z_{C O M}+m \cdot z_{\mathrm{COM}_{L}}}{B M+m}
\end{aligned}
$$

where, $B M$ is the subject body mass, $m$ is the load mass.

For each load carrying way, a one-way analyses of variance (ANOVA) investigating the effect of weight of load on the peak traction coefficient was conducted. The statistical significant differences between the mean values of peak traction coefficient for different weights of the load were found when the significance probability ( $p$-value) was less than $5 \%$, i.e., $p<0.05$.

\section{Results}

Figure 3 shows a typical time change in normal $\left(F_{n}\right)$, horizontal components $\left(F_{h}\right)$ and traction coefficient $\left(\left|F_{h} / F_{n}\right|\right)$ in stance phase (from heel contact to toe off) during walking without carrying loads. It can be seen in Fig. 3, traction coefficient takes the maximum peak values $\left|F_{h} / F_{n}\right|_{h}$ and $\left|F_{h} / F_{n}\right|_{t}$ at heel-strike and toe-off periods, respectively. When the static friction coefficient in the contact interface between a shoe sole and a walkway surface is greater than these two peak values of traction coefficient, slip doesn't occur. Thus, a gait with smaller $\left|F_{h} / F_{n}\right|_{h}$ and $\left|F_{h} / F_{n}\right|_{t}$ is desirable and will reduce the chance of slipping. On the other hand, higher peak traction coefficients will increase the chance of slipping. Meanwhile very large peak values of traction coefficient were usually obtained immediately after heel contact or just before toe-off as shown in Fig. 3, this peak value is anomalous and doesn't cause a macroscopic slip contributing falling. Thus, such anomalous peak traction coefficient was not used in the determination of the $\left|F_{h} / F_{n}\right|_{h}$ and $\left|F_{h} / F_{n}\right| t$.

Figure 4 shows the relationship between weight of load and the peak traction coefficient $\left|F_{h} / F_{n}\right|_{h}$ at heel-strike period. Each plot is mean value of $\left|F_{h} / F_{n}\right|_{h}$, error bar is standard deviation. For 2-handed load carrying and back-pack load carrying, $\left|F_{h} / F_{n}\right|_{h}$ was not affected significantly by weight of load $((F(4,145)=1.583, p=0.181$ for 2 -handed load carrying $)$, $(F(4,145)=0.820, p=0.515$ for back-pack load carrying $))$. On the other hand for waist level front load carrying, $\left|F_{h} / F_{n}\right|_{h}$ was affected significantly by the weight of load $(F(4,145)=9.067, \quad p<0.01), \quad$ and it decreased with increasing of weight of load. These results indicated that it would be effective to reduce the chance of slipping at
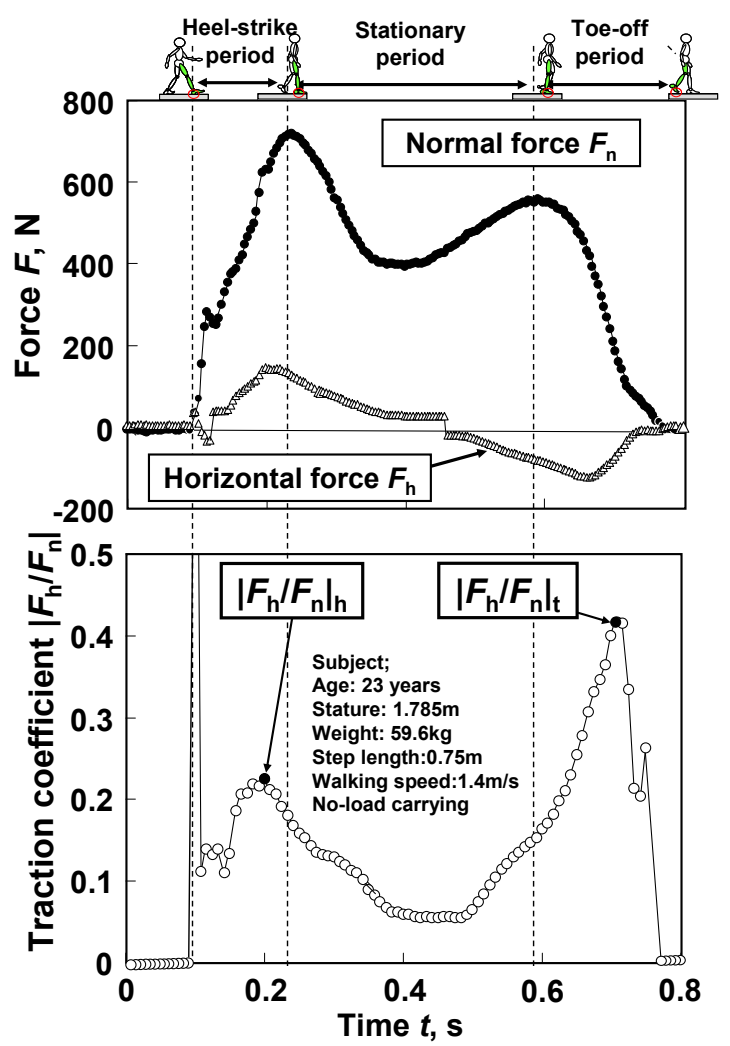

Fig. 3 Typical time change in normal and horizontal components of ground reaction force, and traction coefficient

heel-strike period by carrying loads at front waist level as compared with walking without loads.

Figure 5 shows the relationship between weight of load and the peak traction coefficient $\left|F_{h} / F_{n}\right|_{t}$ at toe-off period. Each plot is mean value of $\left|F_{h} / F_{h}\right|_{h}$, error bar is standard deviation. A one-way ANOVA indicated that $\left|F_{h} / F_{n}\right|_{t}$ for 2-handed load carrying and waist level front load carrying was not affected significantly by weight of load $((F(4,145)=2.357, \quad p=0.056$ for 2-handed load carrying $),(F(4,145)=2.392, p=0.053$ for waist level front load carrying)) and $\left|F_{h} / F_{n}\right|_{t}$ for back-pack load carrying was affected significantly by weight of load $(F(4,145)=4.98, p<0.01)$. However, mean $\left|F_{h} / F_{n}\right|_{t}$ for each way of load carrying has tendency to increase with increasing of weight of load. Thus, it would be considered that the chance of slipping during walking with carrying loads in the way used in this study will be higher than that without carrying loads.

\section{Discussion}

The effects of load carrying on the maximum peak value of traction coefficient at heel-strike period $\left|F_{h} / F_{n}\right|_{h}$ are discussed based on the change in the position of body center of mass in this section. Figure 6 shows the three dimensional geometric relationship between COM or $\mathrm{COM}_{\mathrm{i}}$ and COP. $\theta$ in Fig. 6 is an angle between the vertical line through $\mathrm{COM}_{\mathrm{i}}$ or $\mathrm{COM}$ and the line through 


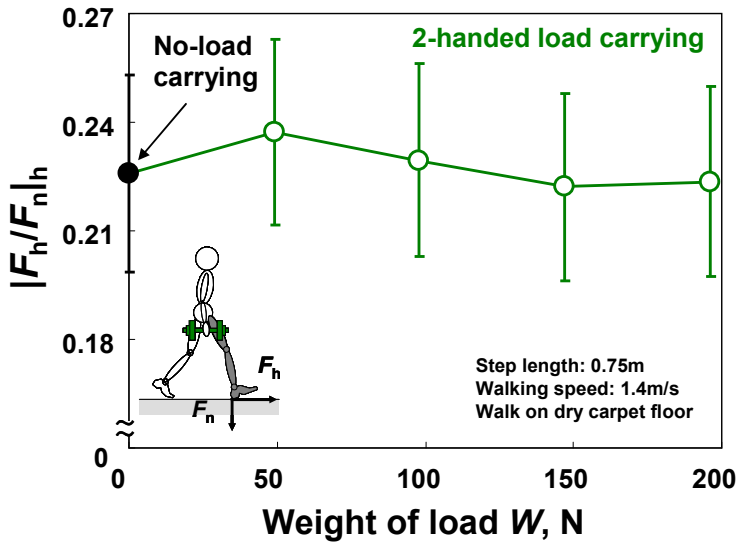

(a) 2-handed load carrying

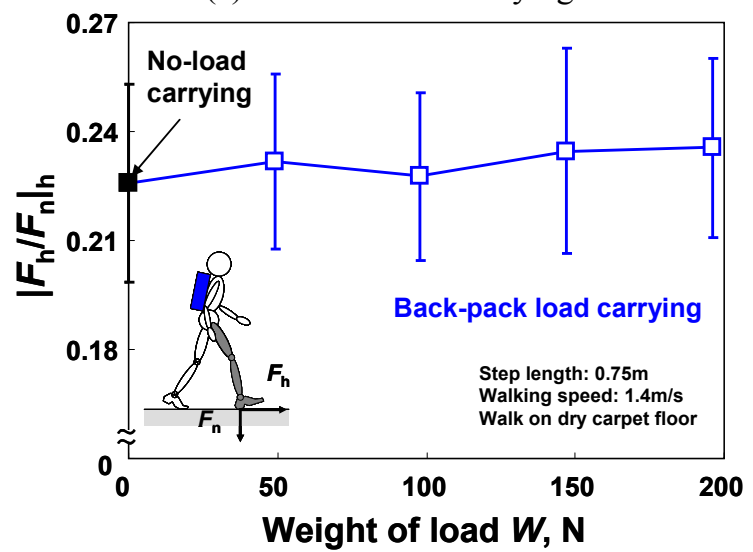

(b) Back-pack load carrying

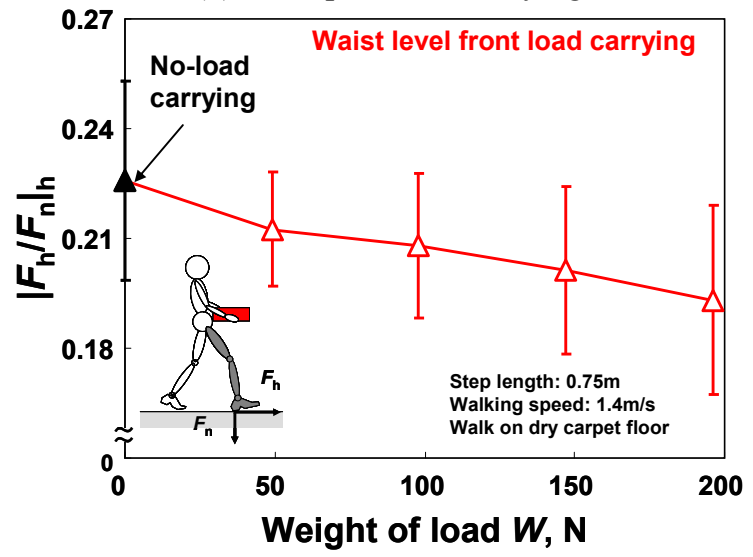

(c) Waist level front load carrying

Fig. 4 The relationship between weight of load and the peak traction coefficient $\left|F_{h} / F_{n}\right|_{h}$

$\mathrm{COM}$ or $\mathrm{COM}_{\mathrm{i}}$ and COP. Based on these geometric relationship between COM $\left(x_{C O M}, y_{C O M}, z_{C O M}\right)$ or $\mathrm{COM}_{1}$ $\left(x_{\mathrm{COMi}}, y_{\mathrm{COM} i}, z_{\mathrm{COM}}\right)$ and the foot $\mathrm{COP}\left(x_{\mathrm{COP}}, y_{\mathrm{COP}}, 0\right)$, $\tan |\theta|$ is expressed by the following equation;

$\tan |\theta|=\left|\frac{l}{h}\right|=\frac{\sqrt{\left(x_{C O P}-x_{C O M}\right)^{2}+\left(y_{C O P}-y_{C O M}\right)^{2}}}{z_{C O M}}$

for walk without carrying loads

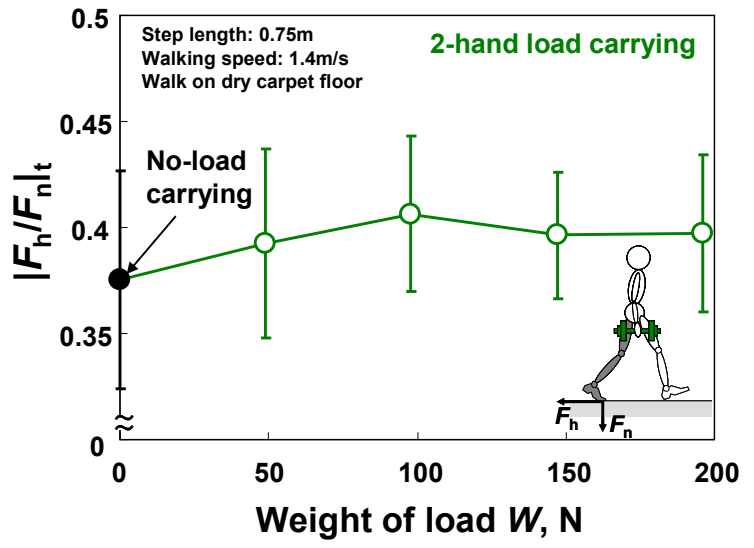

(a) 2-handed load carrying

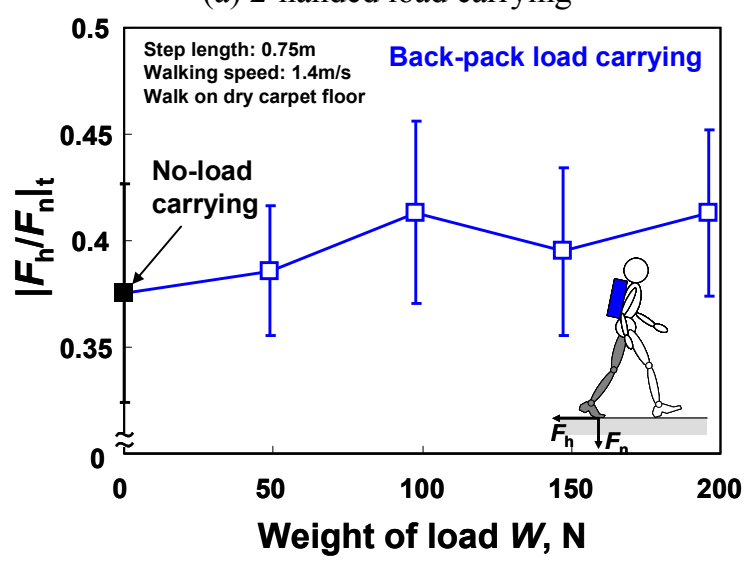

(b) Back-pack load carrying

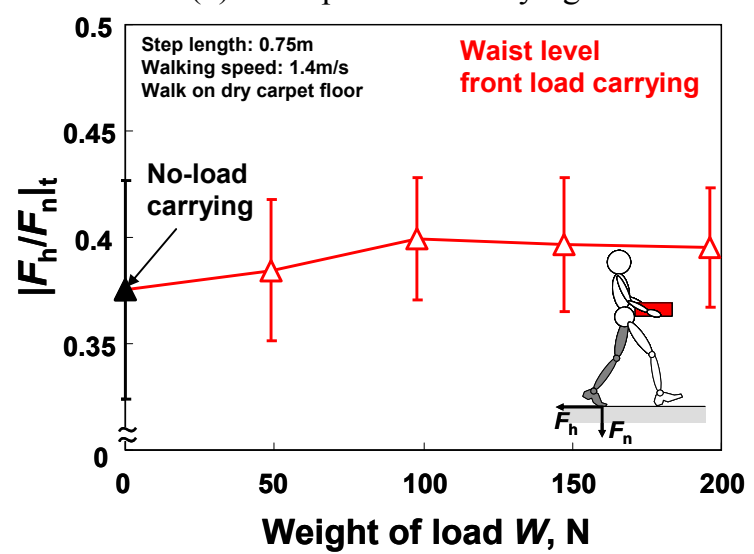

(c) Waist level front load carrying

Fig. 5 The relationship between weight of load and the peak traction coefficient $\left|F_{h} / F_{n}\right|_{t}$

$\tan |\theta|=\left|\frac{l}{h}\right|=\frac{\sqrt{\left(x_{\mathrm{COP}}-x_{\mathrm{COM} i}\right)^{2}+\left(y_{\mathrm{COP}}-y_{\mathrm{COM}_{i}}\right)^{2}}}{z_{\mathrm{COM} i}}$

for walk with carrying loads

where, $l$ is the distance of the COM or $\mathrm{COM}_{\mathrm{i}}$ projected point $\mathrm{P}$ to $\mathrm{COP}, h$ is the height of $\mathrm{COM}$ or $\mathrm{COM}_{\mathrm{i}}$ from $\mathrm{x}-\mathrm{y}$ plane. 


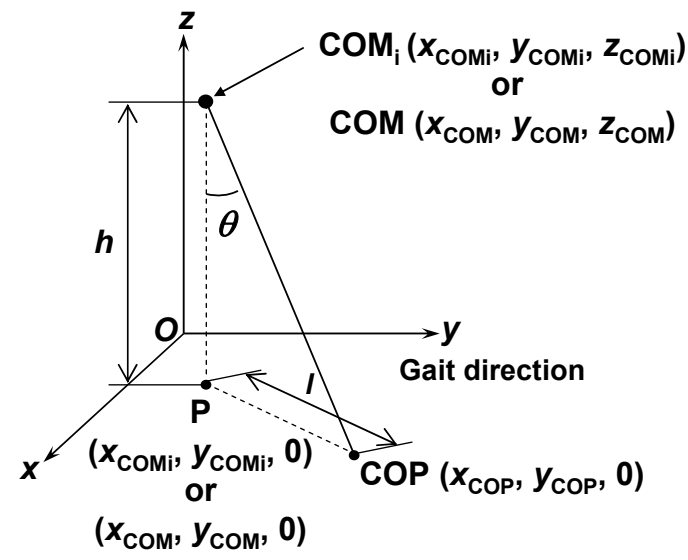

Fig. 6 Three dimensional geometric relationship between $\mathrm{COM}$ or $\mathrm{COM}_{\mathrm{i}}$ and the foot COP

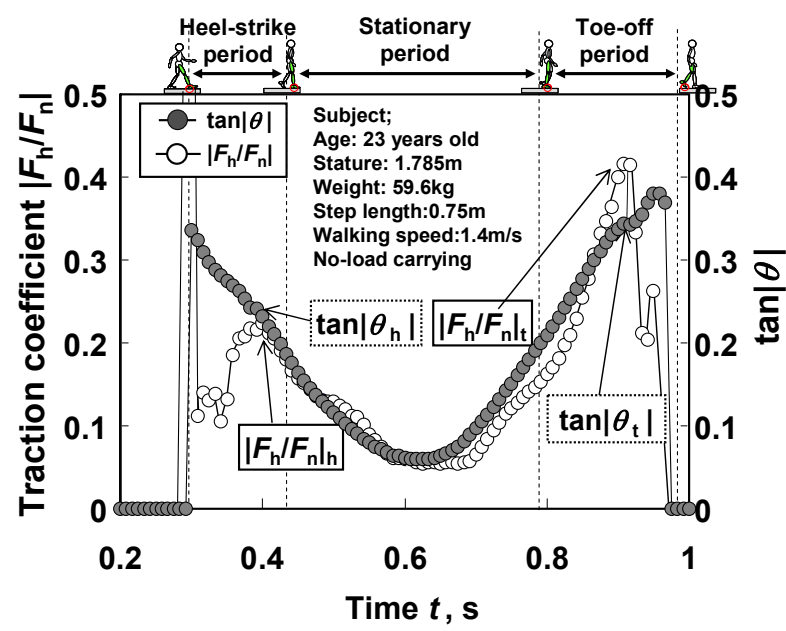

Fig. 7 A typical time change in traction coefficient $\left|F_{h} / F_{n}\right|$ and $\tan |\theta|$ in stance phase for a subject walking without carrying loads

Figure 7 shows a typical time change in traction coefficient $\left|F_{h} / F_{n}\right|$ and $\tan |\theta|$ in stance phase for a subject walking without carrying loads. As shown in Fig. 7, the $\tan \left|\theta_{h}\right|$ is a value of $\tan |\theta|$ at a moment when traction coefficient takes the peak value of $\left|F_{h} / F_{n}\right|_{h}$.

Figure 8 shows the relationship between $\tan \left|\theta_{h}\right|$ and $\left|F_{h} / F_{n}\right|_{h}$ for walk without carrying loads and for walk with carrying loads. As shown in Fig. $8, \tan \left|\theta_{h}\right|$ is positively correlated with $\left|F_{h} / F_{n}\right|_{h}$ for each case, which indicates that $\left|F_{h} / F_{n}\right|_{h}$ can be determined by the $\theta_{h}$ even while walking with carrying loads. It can be seen in Fig. 8(a) and (b) that $\tan \left|\theta_{h}\right|$ for 2-handed and back-pack load carrying takes almost same values as that for walk without carrying loads. Thus, $\left|F_{h} / F_{n}\right|_{h}$ for 2 -handed load carrying or back-pack load carrying was not affected significantly by weight of load. On other hand, it can be seen in Fig. 8(c) that $\tan \left|\theta_{h}\right|$ for a waist level front load carrying takes smaller value than that for walk without carrying loads. Thus, $\left|F_{h} / F_{n}\right|_{h}$ for a waist level load carrying was smaller than that for walk without carrying loads.

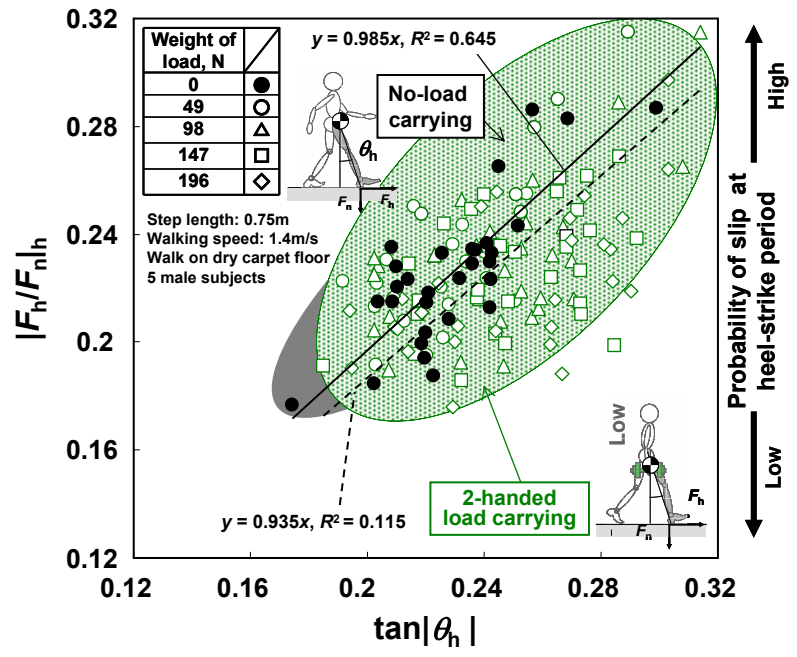

(a) No load carrying and 2-handed load carrying

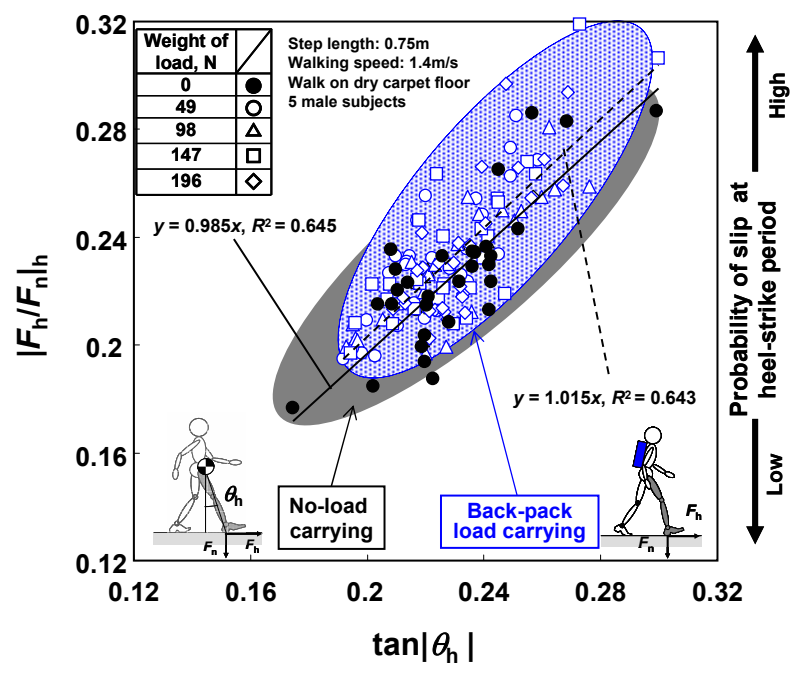

(b) No load carrying and back-pack load carrying

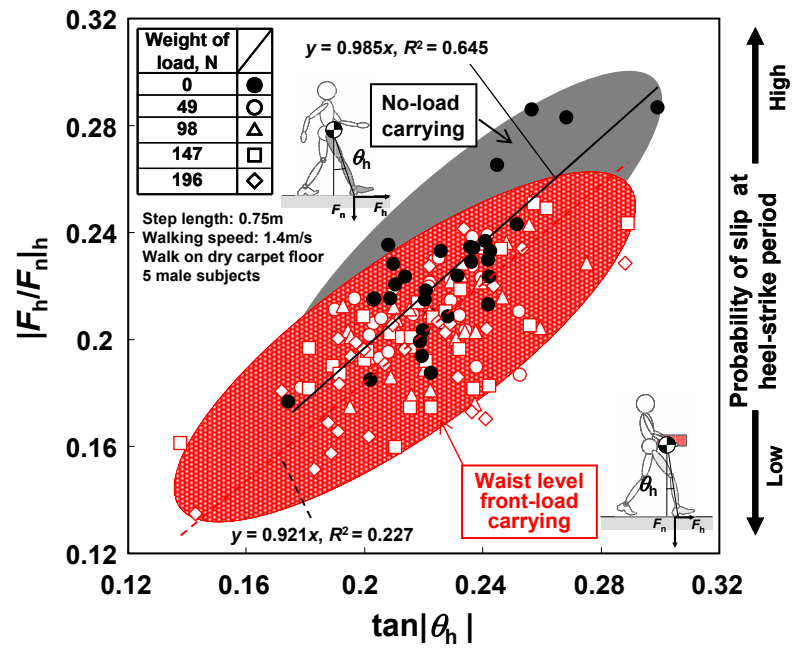

(c) No load carrying and waist level front load carrying

Fig. 8 Relationship between $\tan \left|\theta_{h}\right|$ and $\left|F_{h} / F_{n}\right|_{h}$ for walk without carrying loads and for walk with carrying loads 


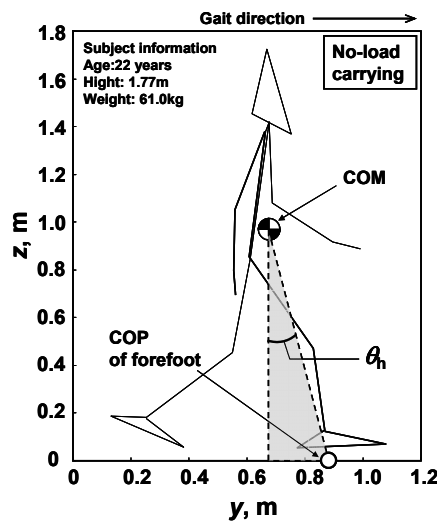

(a)

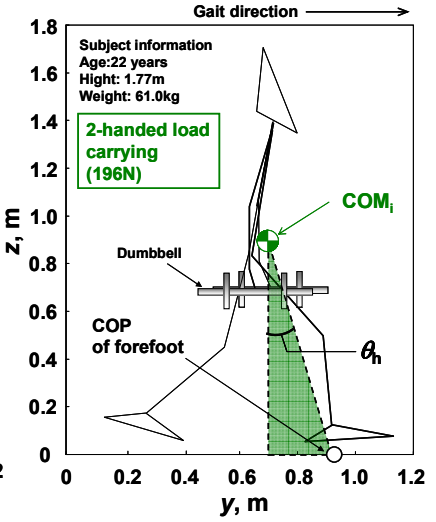

(b)

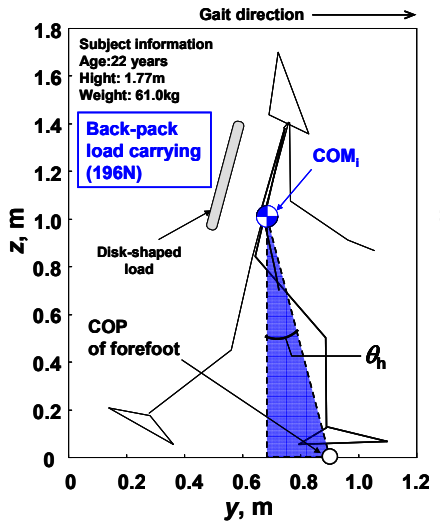

(c)

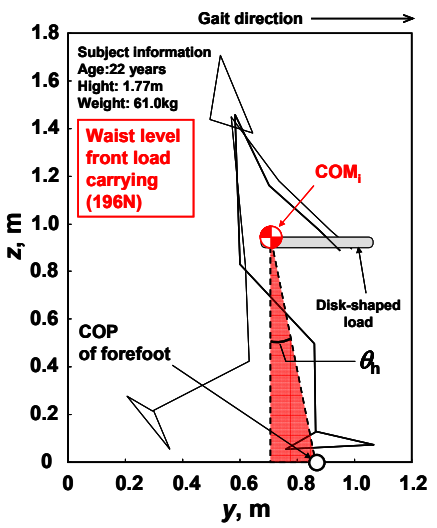

(d)

Fig. 9 Stick-diagrams in sagittal plane when the traction coefficient took the maximum peak value at heel-strike period for (a) walk without carrying loads, (b) 2-handed load carrying, (c) back-pack load carrying, and (d) waist level front load carrying

Figure 9 shows stick-diagrams in sagittal plane when the traction coefficient $\left|F_{h} / F_{n}\right|$ took $\left|F_{h} / F_{n}\right|_{h}$ for walk with loads and without load. It can be seen in Fig. 9(b) that the position of $\mathrm{COM}_{\mathrm{i}}$ for 2-handed load carrying was located slightly inferior to that of COM for walk without load carrying, which would be due to inferior load position. It can also be seen in Fig. 9(c) that the position of $\mathrm{COM}_{\mathrm{i}}$ for back-pack load carrying was located superiorly and posteriorly to that of COM for walk without carrying load, which would be due to superior and posterior position of load. Therefore, the angle $\theta_{h}$ for these two load carrying ways were not significantly different from that for walk without carrying loads.

On the other hand, it can be seen in Fig. 9(d) that the position of $\mathrm{COM}_{\mathrm{i}}$ for waist level front load carrying was located at same height in the vertical direction but significantly anterior to that of COM for walk without carrying loads. Such position change of the body center of mass reduced $\theta_{h}$, which caused smaller $\left|F_{h} / F_{n}\right|_{h}$ than that for no-load condition.

These analyses suggest that it will be effective to reduce $\theta_{h}$ by displacing the loads more anteriorly and more superiorly in order to reduce $\left|F_{h} / F_{n}\right|_{h}$, i.e. the chance of slipping, at heel-strike period.

\section{Conclusions}

The effects of load carrying on the maximum peak values of traction coefficient at heel-strike and toe off periods, i.e. the chance of slipping during walking, were investigated by conducting the gait experiments and kinematic analyses in this paper. The conclusions obtained in this study are summarized as follows;

(1) For waist level front load carrying, $\left|F_{h} / F_{n}\right|_{h}$ was affected significantly by the weight of load and it decreased with increase of weight of load. On the other hand for 2-handed load carrying and back-pack load carrying, $\left|F_{h} / F_{n}\right|_{h}$ was not affected significantly by weight of load.
(2) For each way of load carrying, $\left|F_{h} / F_{n}\right|_{t}$ had tendency to increase with increase of weight of load, which indicates that the chance of slipping during walking with carrying loads in the way used in this study will be higher than that without loads.

(3) It will be effective to reduce $\theta_{h}$ by displacing the loads more anteriorly and more superiorly in order to reduce $\left|F_{h} / F_{n}\right|_{h}$, i.e. the chance of slipping, at heel-strike period.

\section{Acknowledgement}

The authors are grateful to Yasushi Ohta for his assistance in conducting gait experiments.

\section{References}

[1] Minister's Secretariat Statistics and Information Department of Ministry of Health, Labor and Welfare ed., "Vital Statistics of Japan 2004," 1, Health \& Welfare Statistics Association, 2006.

[2] Anderson, R. and Lagerlof, E., "Accident Data in the New Swedish Information System on Occupational Injuries," Ergonomics, 26, 1983, 33-42.

[3] Cham, R. and Redfern, S. M., "Gait Adaptations during Load Carrying on Level and Inclined Surfaces," Occupational Ergonomics, 4, 2004, 11-26.

[4] Yamaguchi, T., Hatanaka, S. and Hokkirigawa, K., "Effect of Step Length and Walking Speed on Traction Coefficient and Slip between Shoe Sole and Walkway," Tribology Online, 3, 2, 2008, 59-64.

[5] Sekiya, N., Nagasaki, H. and Ito, H., "The Invariant Relationship between Step Length and Step Rate during Free Walking," Journal of Human Movement Studies, 30, 1996, 241-257.

[6] Ae, M., Tang, H-P. and Yokoi, T., "Estimation of Inertia Properties of the Body Segment in Japanese Athlete," Journal of the Society of Biomechanism, 11, 1992, 23-33. (in Japanese) 